\title{
Associations Between Sleep Quality, Household Income and Medication Adherence in Adolescents With Asthma
}

\author{
Emily Bryant, Andrea Fidler, \& David Fedele \\ College of Public Health \& Health Professions
}

Faculty Mentor: David Fedele, Department of Clinical and Health Psychology

\begin{abstract}
Adolescents with asthma are at-risk for poor sleep quality. Little research has examined the relationship between sleep quality and inhaled corticosteroids (ICS) adherence. Individuals from lower-income backgrounds experience disparities that may also contribute to sleep quality. This study sought to 1) examine associations between household income and sleep quality and 2) analyze the relationship between sleep quality and ICS adherence among adolescents with asthma. Forty-one adolescents with persistent asthma (Mage $=14.83, \mathrm{SD}=1.28$ ) completed the Adolescent Sleep Wake Scale (ASWS) to measure sleep quality across five subscales. Caregivers provided their estimated household income per year and completed a free response question measuring ICS adherence. Spearman's rank-order correlations were used to examine associations between sleep quality, household income and ICS adherence. Sleep quality and household income were not significantly related $(r=.262, p=.098)$. Sleep quality was not associated with ICS adherence $(r=-.028, \mathrm{p}=.867)$. Sleep quality was not significantly related to household income or ICS adherence in adolescents with asthma. Limitations include limited power given a small sample size and less reliable measures of household income and adherence therefore, future directions should focus on using more precise measures. Additional research with larger sample sizes may help better understand potential relationships between sleep quality, ICS adherence, and household income within youth with asthma.
\end{abstract}

Keywords: adherence, asthma, health disparities, income, sleep

\section{Introduction}

In the United States alone, over 6 million children are living with asthma (Zahran et al., 2018). Asthma is a chronic illness that inflames airways in the lungs, making it difficult to breathe. It is one of the most common chronic diseases in children, and the global prevalence of symptoms continues to increase (Ferrante \& La Grutta, 2018). Asthma management is multidimensional and consists of ongoing education, medication adherence, symptom monitoring, primary care, trigger avoidance, and personalized action plans (Gibson et al., 2003). Many people with asthma use medications such as inhaled corticosteroids (ICS) to manage asthma. Adhering to the proper amount of medication has shown to lead to decreases in 
inflammation of the lungs (Burgess et al., 2010). Unfortunately, barriers often prevent proper ICS adherence from being achieved, as research has shown adherence rates of less than $70 \%$ and in certain populations like low-income adolescents, even lower rates around 33-41\% (McQuaid, 2018). Consequences of poor adherence can result in increased morbidity, exacerbations, hospitalizations and overall lead to poor quality of life for adolescents with asthma and burden on families (Bourdin et al., 2012).

Barriers to ICS adherence can occur at an individual/ familial level, patient-physician level and broader system level (McQuaid, 2018). ICS adherence rates tend to decline as children become older, as a shift in increased responsibilities with asthma management is put onto adolescents (Koster et al., 2015). Individual barriers can include mental health symptoms like depression, which has been found to be an increased risk to poor ICS adherence; other factors like medication beliefs or preference to alternative medicines create barriers for medication adherence, especially for minority groups who may have concerns about historical unethical medical care among diverse minority populations (McQuaid, 2018). Socioeconomically disadvantaged families can experience barriers to accessing consistent asthma care and medications (Canino et al., 2009). Furthermore, lower levels of education may impact one's ability to understand health information about medications and make informed health decisions.

Sleep plays an essential role in the health and well-being of individuals. It is a biological necessity of life, and loss of sleep has been proven to influence higher risks of various infectious and inflammatory diseases as well as mortality (Irwin, 2015). Sleep quality is a concept within the field of psychology that is difficult to define. Ohayon et al. (2017) defines sleep quality as a solicited self-rating reflecting "an individual's satisfaction with his or her sleep" (p. 7). Factors of sleep quality that can be measured include the ability to fall/stay asleep, how rested one feels when they wake up, and the number of awakenings throughout the night. In a sleep study consisting of 943 adolescents, $10 \%$ of the sample reported difficulties falling asleep while $25 \%$ reported needing additional sleep (Morrison et al., 1992). The same study also concluded adolescents with sleeping problems displayed more anxious, depressed, inattentive, and conduct disorder behaviors compared to adolescents without sleeping problems, stressing the importance of sleep within this population. Insufficient sleep within adolescent age groups has been associated with poor physical and mental health outcomes, academic outcomes, and neurocognitive deficits (Mindell et al., 2016). 
Adolescents with asthma appear to experience more sleep problems than their healthy counterparts. In a study comparing sleep duration, sleep hygiene and insomnia in adolescents with and without asthma, the results found adolescents with asthma reported insufficient weekday sleep $13 \%$ higher than those without asthma, a significant difference in poor sleep hygiene for adolescents with severe asthma, and twice as many adolescents with severe asthma reporting clinically significant insomnia compared to participants with mild or no asthma (Meltzer et al., 2014). Some factors that create this gap between adolescents with asthma and their healthy counterparts include nocturnal symptoms (e.g., coughing, wheezing, shortness of breath) lower sleep duration, and nighttime awakenings (Lawless et al., 2020). This places adolescents with asthma at an increased risk for poor sleep which can lead to additional negative outcomes.

Poor asthma control has been associated with sleep quality, as seen in Luyster et al. (2012), which found people with asthma experience worse sleep quality than those without asthma, and poor sleep quality is associated with poor asthma control. However, little research has been conducted on how sleep quality may impact ICS adherence in adolescents with asthma. To date, there is one study that found an indirect, significant effect of sleeping problems on medication usage through compromised family functioning, specifically behavioral control (Sonney et al., 2017). No direct associations were found. No other study has examined the direct associations between sleep quality and asthma ICS adherence, highlighting a gap in the literature within this population.

In addition to asthma symptoms, socioeconomic (SES) factors like household income may influence sleep quality in this population. This may be due to economic disparities preventing access to resources like adequate bedding, and neighborhood environments which lower-income households experience such as higher exposure to light, noise, and overcrowded housing conditions (Mindell et al., 2016). Of the studies conducted, there are mixed results between sleep quality and household income levels. A systematic review of eight included studies suggested poorer neighborhood socioeconomic status was associated with shorter sleep duration in children (Tomfohr-Madsen et al., 2020). One of the studies highlighted in the review used an income-toneeds ratio to examine if presleep worries and home environment conditions were mediators between low SES and poor sleep (Bagley et al., 2015). The results found lower SES was associated with more sleep/wake issues and daytime sleepiness, mediated by disruptive 
conditions and presleep worries (Bagley et al., 2015). Contrary to these findings, Smaldone et al. (2007) examined characteristics related to inadequate sleep within children age 6-11 and adolescents age 12-17. The results showed children with inadequate sleep were more frequent among non-Hispanic white children whose families have higher incomes and education levels. The mixed results displayed throughout the current literature require further investigation.

\section{Aims and Hypotheses}

For youth with asthma, multiple factors can contribute to significant effects on health outcomes, such as relationships between poor sleep quality, nonadherence to ICS controllers and demographic variables. Little research has been conducted on sleep quality and the potential impacts it has on ICS adherence, while the literature on sleep quality and household income contains mixed results. This project aims to 1) examine the associations between household income and sleep quality in adolescents and 2) examine the relationship between sleep quality and ICS adherence for adolescents with asthma. For aim 1, it is hypothesized household income will be correlated with sleep quality, with lower income being related to poorer sleep quality. This is based on the prior meta-analysis of neighborhood socioeconomic status and sleep duration that included studies of poor environmental conditions which low-income adolescents are exposed to (Tomfohr-Madsen et al., 2020). For aim 2, it is hypothesized sleep quality will be associated with ICS adherence for adolescents with asthma, with poorer sleep quality being related to lower ICS adherence. Reasoning behind this includes findings on the relationship poor asthma control has on sleep quality (Luyster et al., 2012). It is important to continue research efforts on health disparities in order to create targeted interventions that prevent morbidity and mortality, provide better healthcare services, reduce disparities and promote health for families with lower SES. Research efforts to understand more about the relationship between sleep quality and ICS adherence may lead to the development of more targeted interventions aimed at improving health outcomes among adolescents with asthma, such as increasing educational efforts on how to promote healthy sleep habits, understanding the relationship between sleep quality and asthma, and how to better adhere to controller medication.

\section{Methods}

\section{Participants}

Participants consisted of 41 adolescents, aged 13-17 with persistent asthma, and their parents/ legal guardians. Participants were included if they 1) had persistent asthma, 2) were between 
ages $13-17,3$ ) had a parent/legal guardian accompany the child to the appointment, and 4) were attending a scheduled visit at UF's pediatric clinic. Exclusion criteria consisted of 1) the inability to read and speak English and 2) having an intellectual disability diagnosis or psychotic disorder.

\section{Procedures}

Adolescents were recruited from the Pediatric Pulmonary Diagnostic Center in the Medical Plaza of UF Health. The parent/ legal guardian provided informed consent while the adolescent gave informed assent prior to completing the study. The adolescent and caregiver completed study questionnaires during their clinic visit and were compensated for their participation. The institutional review board approved this study (IRB201300732). This study is based on secondary analyses from a previously published study analyzing sleep hygiene and the potential relationships between sleep quality, cognitive fatigue, asthma control and management, and quality of life in adolescents with asthma (Lawless et al., 2020).

\section{Measures}

\section{demographics.}

Caregivers completed demographic information including the adolescent's date of birth, sex, race, ethnicity, grade level in school, in addition to their own age, household income, occupation, educational level, and marital status. Caregivers identified the estimated family income per year through the options “below \$9,999," “\$10,000-\$19,999," “\$20,000-29,999," “\$30,000-\$39,999," “\$40,000-\$49,999,” “\$50,000-\$59,999,” “\$60,000-\$69,999,” “\$70,000-\$79,999,” and “over $\$ 80,000$."

\section{sleep quality.}

Adolescents completed the Adolescent Sleep Wake Scale (ASWS) (LeBourgeois et al., 2005), a 28-item measure which assessed sleep quality within the past month across the following sub-scales: going to bed, falling asleep, maintaining sleep, reinitiating sleep, and returning to wakefulness. Overall sleep quality was measured by calculating the average of the sub-scale scores. The measure addressed the frequency of sleep behaviors that occurred during the last month. Items used a 6-point Likert scale, where $1=$ "always" and $6=$ "never. Higher scores indicate better sleep quality. 


\section{medication adherence.}

Caregivers completed a single free response question which measured medication adherence. The question asked, "In the last 30 days, how many days do you estimate that your child took his/her asthma controller medication(s) exactly as prescribed?"

\section{Analyses}

Analyses was conducted in SPSS. The data was examined for normality. Basic descriptive analyses were ran to characterize the sample. Statistical significance was analyzed at $(\mathrm{p}<.05)$. Cohen's (1992) conventions were used to describe the correlation coefficient (r). A correlation coefficient of 0.1 indicates a small effect size, 0.3 a medium effect size and 0.5 a large effect size. Spearman's rank-order correlations were conducted to examine associations between household income and sleep quality. Partial Spearman rank-order correlations were conducted to assess if sleep quality related to asthma medication adherence. The variable age was controlled for during data analysis due to known associations between age and sleep quality in addition to medication adherence.

\section{Results}

Initial analysis included checking for skewness and kurtosis through generating histograms and running descriptive statistics. ICS adherence was highly skewed, with over a third of families reporting 100\% ICS adherence. The study used Spearman's Rank-Order Correlations to account for the uneven distributions. Participants ranged from 13 to 17 years $(M=14.83$, $\mathrm{SD}=1.28)$. ICS adherence ranged from 0 to 30 days $(\mathrm{M}=20.65, \mathrm{SD}=10.90)$. Of the 41 adolescents, 21 were male. The majority of caregivers were currently married $(n=18)$, had completed some college $(n=20)$, and consisted of mothers $(n=36)$. Income ranged from below $\$ 9,999$ to over $\$ 80,000$, with the largest percentage of families generating $\$ 10,000$ to $\$ 19,999$ per year $(29.3 \%)$. Table 1 provides additional demographic information. 
Table 1. Participant and Caregiver Demographic Characteristics

\begin{tabular}{lcc}
\hline & Frequency & Percent \\
\hline Participant Gender & 21 & 51.2 \\
$\quad$ Male & 20 & 48.8 \\
$\quad$ Female & & \\
Caregiver Relationship & 36 & 87.8 \\
$\quad$ Mother & 4 & 9.8 \\
Father & 1 & 2.4 \\
Other Legal Guardian & & \\
Caregiver Marital Status & 18 & 43.9 \\
$\quad$ Currently Married & 12 & 29.3 \\
Single, Divorced & 7 & 17.1 \\
Single, Never Married & 3 & 7.3 \\
Single, Co-Habitatiog & 1 & 2.4 \\
Single, Widowed & 1 & \\
Caregiver Education Completed & 3 & 2.4 \\
Middle school & 7 & 7.3 \\
Some high school & 20 & 17.1 \\
Graduated high school & 9 & 48.8 \\
Some college & 1 & 22.0 \\
Graduated college & & 2.4 \\
Post-Graduate school & 5 & 12.2 \\
Family Income & 12 & 29.3 \\
Below \$9,999 & 4 & 9.8 \\
\$10,000-\$19,999 & 5 & 12.2 \\
\$20,000-\$29,999 & 7 & 17.1 \\
\$30,000-\$39,999 & 1 & 2.4 \\
\$40,000-\$49,999 & 1 & 2.4 \\
\$50,000-\$59,999 & 4 & 9.8 \\
\$60,000-\$69,999 & 2 & 4.9 \\
\$70,000-\$79,999 & & \\
Over \$80,000 & & \\
\hline & &
\end{tabular}

For aim 1, there was not a significant relationship between overall sleep quality and household income ( $\mathrm{r}=.262, \mathrm{p}=.098$ ). The effect size was small as described by Cohen's 1992 conventions. None of the ASWS sub scales were significantly related to income. For aim 2, sleep quality was not associated with ICS adherence $(\mathrm{r}=-.028, \mathrm{p}=.867)$. Table 2 shows additional Spearman's Rank-Order Correlations between the ASWS subscales, household income, and ICS adherence.

Table 2. Spearman's Rank-Order Correlations

\begin{tabular}{lllll}
\hline & ICS Adherence & & Household Income & \\
\cline { 2 - 5 } & Correlation coefficient & Sig. (2-tailed) & Correlation coefficient & Sig. (2-tailed) \\
\hline Total Sleep Quality & -.028 & -.867 & .262 & .098 \\
Returning to & -.105 & .524 & .173 & .279 \\
Wakefulness & & & & .249 \\
Reinitiating Sleep & .017 & .916 & .287 & .069 \\
Maintaining Sleep & .073 & .660 & .183 & .251 \\
Falling Asleep & -.080 & .627 & .182 & .254 \\
Going to Bed & .000 & 1.000 & & .254 \\
\hline
\end{tabular}

\section{Discussion}

Adolescents with asthma face an increased risk of poor sleep and negative health outcomes. For those living in low-income households, they may face additional disparities surrounding asthma prevalence (Akinbami et al., 2012). This study examined associations between 1) household income and sleep quality and 2) ICS adherence and sleep quality in adolescents with 
asthma. Overall, no significant associations were found between sleep quality, income, and ICS adherence. The results in this study are important in further understanding relationships between sleep quality and household income in adolescents with asthma and aids in advancing clearer directions in research on this population.

The hypothesis in which lower household income would relate to poorer sleep quality was not supported, despite there being some prior literature suggesting socioeconomic status was associated with reduced sleep duration and quality (Bagley et al., 2015; Felden et al., 2015; Tomfohr-Madsen et al., 2020). The results from this study do relate to other mixed literature that did not find associations between household income, sleep duration, and sleep quality (Johnson et al., 2018). Additionally, other findings have found higher SES relating to poorer sleep quality (Smaldone et al., 2007). The effect size on overall sleep quality and household income was small, suggesting this finding be re-evaluated within larger samples.

The hypothesis that poorer sleep quality would be associated with lower ICS adherence was not supported. Although literature shows people with poorly controlled asthma appear to experience poorer sleep health (Luyster et al., 2012; Prasad et al., 2014), this study's findings do not suggest poor sleep quality is associated with ICS adherence. Only one known study has examined how sleep quality may impact ICS adherence, and found an indirect significant effect through behavioral control. The study did not find any direct associations between sleep disturbances and adherence and suggests analyzing other factors that may interact between the two variables (Sonney et al., 2017). When examining sleep quality and adherence within other diseases, such as HIV, one study found mediating effects of depressive symptoms influencing the relationship between sleep quality and adherence (Phillips et al., 2005). It may be possible sleep quality indirectly relates to ICS adherence through other unknown factors. One potential variable that would be important to examine is allergic rhinitis, which is a common comorbidity in people with asthma and may relate to sleep disturbances (Dixon et al., 2006). Additional research is suggested.

The current study had several limitations. One limitation was the small sample size. Increasing the sample size may have allowed an increase in statistical power. Another limitation was the chosen measures for household income and ICS adherence. The measure for household income may not adequately represent socioeconomic status because it is multifaceted, including other factors such as educational attainment and occupational status (Adler \& Stewart, 2007). A 
caregiver-report was the only measure of adolescent ICS adherence within the study, which may have been biased and inaccurate based on their perceptions of their adolescent's ICS adherence. Literature suggests self-report measures are subject to bias and over-estimated compared to objective measures (Adams et al., 1999). A study measuring adherence in children with asthma showed on average, self-report measures of adherence were reported above $80 \%$, canister weight measures of adherence were around 69\%, and electronic devices measures of adherence were around $50 \%$, suggesting objective measures of adherence may be more accurate (Bender et al., 2000). Additionally, the sample heavily consisted of Caucasian ( $n=18)$ and African American $(n=17)$ participants. The findings in this study therefore may not be generalizable with other diverse populations in the US.

\section{Conclusion}

Future research should examine the relationship between household income and sleep quality using a more accurate measure of socioeconomic status, such as the MacArthur Sociodemographic questionnaire (Adler \& Stewart, 2007). Future studies may want to analyze specific factors of sleep quality concerning household income, like maintaining sleep. Studies analyzing sleep quality and ICS adherence should use an objective measure of adherence. Additional research is necessary to better understand whether sleep quality is associated with household income and ICS adherence in adolescents with asthma.

The results of this study add to the current literature known about sleep quality in relation to household income and ICS adherence. Although this study revealed null results, it is important to publish null findings to prevent publication bias and prevent retesting of hypotheses shown unlikely be true (Landis et al., 2014; Munafò \& Neill, 2016). The null findings in this study aid to create a more complete body of literature surrounding sleep quality, household income, and ICS adherence in adolescents with asthma, which advances scientific knowledge within this population.

\section{Acknowledgements}

I would like to acknowledge and give thanks to my mentor, David Fedele, for allowing me the opportunity to pursue my own research project and to be a part of his team for the last three years. I would also like to thank Andrea Fidler for guiding me through this project every step of the way and always believing in me. Finally, I would like to thank all the members of the Pediatric Behavioral Health Lab for their support and words of wisdom over the years. 


\section{References}

Adams, A. S., Soumerai, S. B., Lomas, J., \& Ross-Degnan, D. (1999). Evidence of self-report bias in assessing adherence to guidelines. International Journal for Quality in Health Care, 11(3), 187192. https://doi.org/10.1093/intqhe/11.3.187

Adler, N., \& Stewart, J. (2007). The MacArthur scale of subjective social status. San Francisco: MacArthur Research Network on SES \& Health. http://www.macses.ucsf.edu/research/psychosocial/subjective.php

Akinbami, L. J., Moorman, J. E., Bailey, C., Zahran, H. S., King, M., Johnson, C. A., \& Liu, X. (2012). Trends in asthma prevalence, health care use, and mortality in the United States, 2001-2010. NCHS data brief, (94), 1-8.

Bagley, E. J., Kelly, R. J., Buckhalt, J. A., \& El-Sheikh, M. (2015). What keeps low-SES children from sleeping well: the role of presleep worries and sleep environment. Sleep Medicine, 16(4), 496502. https://doi.org/10.1016/j.sleep.2014.10.008

Bender, B., Wamboldt, F., O'Connor, S. L., Rand, C., Szefler, S., Milgrom, H., \& Wamboldt, M. Z. (2000). Measurement of children's asthma medication adherence by self report, mother report, canister weight, and Doser CT. Annals of Allergy, Asthma \& Immunology, 85(5), 416-421. https://doi.org/10.1016/S1081-1206(10)62557-4

Bourdin, A., Halimi, L., Vachier, I., Paganin, F., Lamouroux, A., Gouitaa, M., ... \& Chanez, P. (2012). Adherence in severe asthma. Clinical \& Experimental Allergy, 42(11), 1566-1574. https://doi.org/10.1111/j.1365-2222.2012.04018.x

Burgess, S., Sly, P., \& Devadason, S. (2010). Adherence with preventive medication in childhood asthma. Pulmonary medicine, 2011. https://doi.org/10.1155/2011/973849

Canino, G., McQuaid, E. L., \& Rand, C. S. (2009). Addressing asthma health disparities: a multilevel challenge. Journal of Allergy and Clinical Immunology, 123(6), 1209-1217. https://doi.org/10.1016/j.jaci.2009.02.043

Cohen, J. (1992). A power primer. Psychological bulletin, 112(1), 155-159. https://doi.org/10.1037/00332909.112.1.155

Dixon, A. E., Kaminsky, D. A., Holbrook, J. T., Wise, R. A., Shade, D. M., \& Irvin, C. G. (2006). Allergic rhinitis and sinusitis in asthma: differential effects on symptoms and pulmonary function. Chest, 130(2), 429-435. https://doi.org/10.1378/chest.130.2.429

Felden, É. P. G., Leite, C. R., Rebelatto, C. F., Andrade, R. D., \& Beltrame, T. S. (2015). Sono em adolescentes de diferentes níveis socioeconômicos: revisão sistemática. Revista Paulista de Pediatria, 33(4), 467-473. https://doi.org/10.1016/j.rpped.2015.01.011

Ferrante, G., \& La Grutta, S. (2018). The burden of pediatric asthma. Frontiers in pediatrics, 6, 186. https://doi.org/10.3389/fped.2018.00186

Gibson, P. G., Ram, F. S., \& Powell, H. (2003). Asthma education. Respiratory medicine, 97(9), 10361044. https://doi.org/10.1016/S0954-6111(03)00134-3 
Irwin, M. R. (2015). Why sleep is important for health: a psychoneuroimmunology perspective. Annual review of psychology, 66. https://doi.org/10.1146/annurev-psych-010213-115205

Johnson, D. A., Meltzer, L. J., Zhang, T., Lu, M., Cassidy-Bushrow, A. E., Stokes-Buzzelli, S., ... \& Joseph, C. L. (2018). The influence of psychosocial stressors and socioeconomic status on sleep among caregivers of teenagers with asthma, the Puff City study. Sleep health, 4(2), 141-146. https://doi.org/10.1016/j.sleh.2017.11.003

Koster, E. S., Philbert, D., de Vries, T. W., van Dijk, L., \& Bouvy, M. L. (2015). "I just forget to take it": asthma self-management needs and preferences in adolescents. Journal of Asthma, 52(8), 831837. https://doi.org/10.3109/02770903.2015.1020388

Landis, R. S., James, L. R., Lance, C. E., Pierce, C. A., \& Rogelberg, S. G. (2014). When is nothing something? Editorial for the null results special issue of Journal of Business and Psychology. Journal of Business and Psychology, 29(2), 163-167. https://doi.org/10.1007/s10869-014-9347-8

Lawless, C., Turner, E. M., LeFave, E., Koinis-Mitchell, D., \& Fedele, D. A. (2020). Sleep hygiene in adolescents with asthma. Journal of Asthma, 57(1), 62-70.

https://doi.org/10.1080/02770903.2018.1553049

LeBourgeois, M. K., Giannotti, F., Cortesi, F., Wolfson, A. R., \& Harsh, J. (2005). The relationship between reported sleep quality and sleep hygiene in Italian and American

adolescents. Pediatrics, 115(Supplement 1), 257-265.

https://doi.org/10.1542/peds.2004-0815H

Luyster, F. S., Teodorescu, M., Bleecker, E., Busse, W., Calhoun, W., Castro, M., ... \& Wenzel, S. E. (2012). Sleep quality and asthma control and quality of life in non-severe and severe asthma. Sleep and Breathing, 16(4), 1129-1137. https://doi.org/10.1007/s11325-011-0616-8

McQuaid, E. L. (2018). Barriers to medication adherence in asthma: the importance of culture and context. Annals of Allergy, Asthma \& Immunology, 121(1), 37-42. https://doi.org/10.1016/j.anai.2018.03.024

Meltzer, L. J., Ullrich, M., \& Szefler, S. J. (2014). Sleep duration, sleep hygiene, and insomnia in adolescents with asthma. The Journal of Allergy and Clinical Immunology: In Practice, 2(5), 562-569. https://doi.org/10.1016/j.jaip.2014.02.005

Mindell, J. A., Sedmak, R., Boyle, J. T., Butler, R., \& Williamson, A. A. (2016). Sleep well!: A pilot study of an education campaign to improve sleep of socioeconomically disadvantaged children. Journal of Clinical Sleep Medicine, 12(12), 1593-1599. https://doi.org/10.5664/jcsm.6338

Morrison, D. N., McGee, R. O. B., \& Stanton, W. R. (1992). Sleep problems in adolescence. Journal of the American Academy of Child \& Adolescent Psychiatry, 31(1), 94-99. https://doi.org/10.1097/00004583-199201000-00014

Munafò, M., \& Neill, J. (2016). Null is beautiful: On the importance of publishing null results. Journal of Psychopharmacology, 30(7), 585-585. https://doi.org/10.1177/0269881116638813 
Ohayon, M., Wickwire, E. M., Hirshkowitz, M., Albert, S. M., Avidan, A., Daly, F. J., ... \& Hazen, N. (2017). National Sleep Foundation's sleep quality recommendations: first report. Sleep health, 3(1), 6-19. https://doi.org/10.1016/j.sleh.2016.11.006

Phillips, K. D., Moneyham, L., Murdaugh, C., Boyd, M. R., Tavakoli, A., Jackson, K., \& Vyavaharkar, M. (2005). Sleep disturbance and depression as barriers to adherence. Clinical nursing research, 14(3), 273-293. https://doi.org/10.1177\%2F1054773805275122

Prasad, B., Nyenhuis, S. M., \& Weaver, T. E. (2014). Obstructive sleep apnea and asthma: associations and treatment implications. Sleep medicine reviews, 18(2), 165-171. https://doi.org/10.1016/j.smrv.2013.04.004

Smaldone, A., Honig, J. C., \& Byrne, M. W. (2007). Sleepless in America: inadequate sleep and relationships to health and well-being of our nation's children. Pediatrics, 119(Supplement 1), S29-S37. https://doi.org/10.1542/peds.2006-2089F

Sonney, J. T., Segrin, C., \& Ward, T. M. (2017). Associations among behavioral sleep disturbance, family functioning, and controller medication adherence in children with asthma. Journal of Developmental \& Behavioral Pediatrics, 38(3), 208-214. https://doi.org/10.1097/DBP.0000000000000433

Tomfohr-Madsen, L. M., Cameron, E. E., Dhillon, A., MacKinnon, A., Hernandez, L., Madigan, S., \& Tough, S. (2020). Neighborhood socioeconomic status and child sleep duration: a systematic review and meta-analysis. Sleep Health. https://doi.org/10.1016/j.sleh.2020.02.012

Zahran, H. S., Bailey, C. M., Damon, S. A., Garbe, P. L., \& Breysse, P. N. (2018). Vital signs: asthma in children-United States, 2001-2016. Morbidity and Mortality Weekly Report, 67(5), 149. http://dx.doi.org/10.15585/mmwr.mm6705e1 